\title{
Atypical and clinically silent multiple sclerosis: a report of 12 cases discovered unexpectedly at necropsy
}

\author{
JG PHADKE, PV BEST \\ From the Departments of Neurology and Pathology, Aberdeen Royal Infirmary, Aberdeen, Scotland
}

SUMMARY Twelve cases are reported in which multiple sclerosis was discovered unexpectedly at necropsy. Four of the patients had no known previous history of neurological illness, four did have a previous neurological or neuropsychiatric disturbance, but multiple sclerosis was never entertained in the differential diagnosis and four had a history of disorder in the nervous system, but a diagnosis of multiple sclerosis was either rejected or regarded as unlikely. In six of the eight patients with a previous history of neurological illness the onset was at or over the age of 50 years. Computed tomography and measurement of evoked responses should now reduce the number of undiagnosed cases, but some will inevitably remain, giving rise to an underestimate of disease prevalence.

Clinical diagnosis of multiple sclerosis can be difficult early in the course of the disease, particularly if the onset is after the age of 50 years and when the presentation is atypical. Discovery of the disease unexpectedly at necropsy is nevertheless unusual $^{1-13}$ and we report a series of 12 such cases.

\section{Case Reports}

The cases were divided into three groups: I-patients with no known history of neurological illness; II-patients with a previous history of neurological disturbance, but in whom multiple sclerosis was never considered in the differential diagnosis; III-patients with a history of neurological disorder in whom a diagnosis of multiple sclerosis was rejected or considered unlikely.

Group 1 Cases with no known previous history of neurological illness

For each case in this group, confirmation of the absence of a history of neurological disorder was obtained from the case notes and the general practitioner.

Case 1 A man aged 72 years was admitted as an emergency in acute respiratory failure superimposed on chronic bronchitis. He had no neurological symptoms apart from difficulty in using has left arm due to "frozen shoulder" developed after an injury. He was dyspnoeic and had

Address for reprint requests: Dr PV Best, Department of Pathology, University Medical Buildings, Foresterhill, Aberdeen AB9 2ZD, Scotland.

Received 29 June 1982 and in revised form 24 November 1982 Accepted 9 December 1982 generalised lymphadenopathy with radiographic evidence of mediastinal masses and collapse of the right lower lobe of lung. Other radiographs demonstrated the presence of diffuse metastatic disease. He died within 48 hours of admission. At necropsy there were features of carcinomatosis but the primary could not be identified. In the brain there were scattered, well demarcated grey, gliotic plaques of demyelination in the white matter, mostly paraventricular, although a few were present peripherally. The lesions were typical of multiple sclerosis.

Case 2 A man aged 72 years was admitted as an emergency to the Chest Unit with severe dyspnoea. He had had obstructive airways disease for two years, which had recently worsened. He was a known alcoholic and heavy smoker, living alone, who had neglected himself most of his life. On clinical examination there were signs of advanced cor pulmonale. Neurologically there was no specific abnormality apart from gross generalised muscle wasting, attributable to poor nutrition, and disorientation due to carbon dioxide narcosis. He died on the fourth day with progressive respiratory insufficiency. At necropsy, apart from severe emphysema and changes attributable to diffuse myocardial insufficiency, abnormalities were confined to the central nervous system (CNS). Several small, grey, gliotic plaques, typical of multiple sclerosis, were present beside the posterior horns of the lateral ventricles and in the brainstem.

Case 3 A woman aged 62 years was admitted to a medical ward with headache, vomiting, drowsiness and urinary incontinence of sudden onset. On examination she was found to be hypertensive and had signs of a left spastic hemiplegia with facial involvement. For three months prior to this she had been behaving abnormally. After admission 
she became progressively less responsive and died on the fifth day of hospitalisation. At necropsy, apart from bronchopneumonia and minimal coronary atheroma, abnormalities were confined to the brain. There was a large haematoma in the right occipital region and this was evidently the cause of death. In addition, however, numerous well-demarcated plaques characteristic of multiple sclerosis were found in the white matter around the ventricles, peripherally in the cerebral hemispheres and in the cerebellum and brainstem. The cerebral haemorrhage appeared to have occurred into one of the large patches of demyelination.

Case 4 A man born in 1903 was seen in the Orthopaedic Department in 1967 for pain in the back, stiffness of the left hip and tingling on the anterior aspect of the left leg. A diagnosis of osteoarthritis of the left hip and lumbo-sacral region was made. No neurological abnormality was detected. In 1977 he had a perforated duodenal ulcer treated surgically, when again no neurological abnormality was noted. In 1980 his family doctor saw him for the treatment of congestive cardiac failure and he did not notice any neurological abnormality. Until a month before his death, he was able to walk with a stick and the difficulty he had had in walking was ascribed to osteoarthritis of the hips. In 1982 he was admitted with streptococcal septicaemia from unknown source. He was very drowsy and

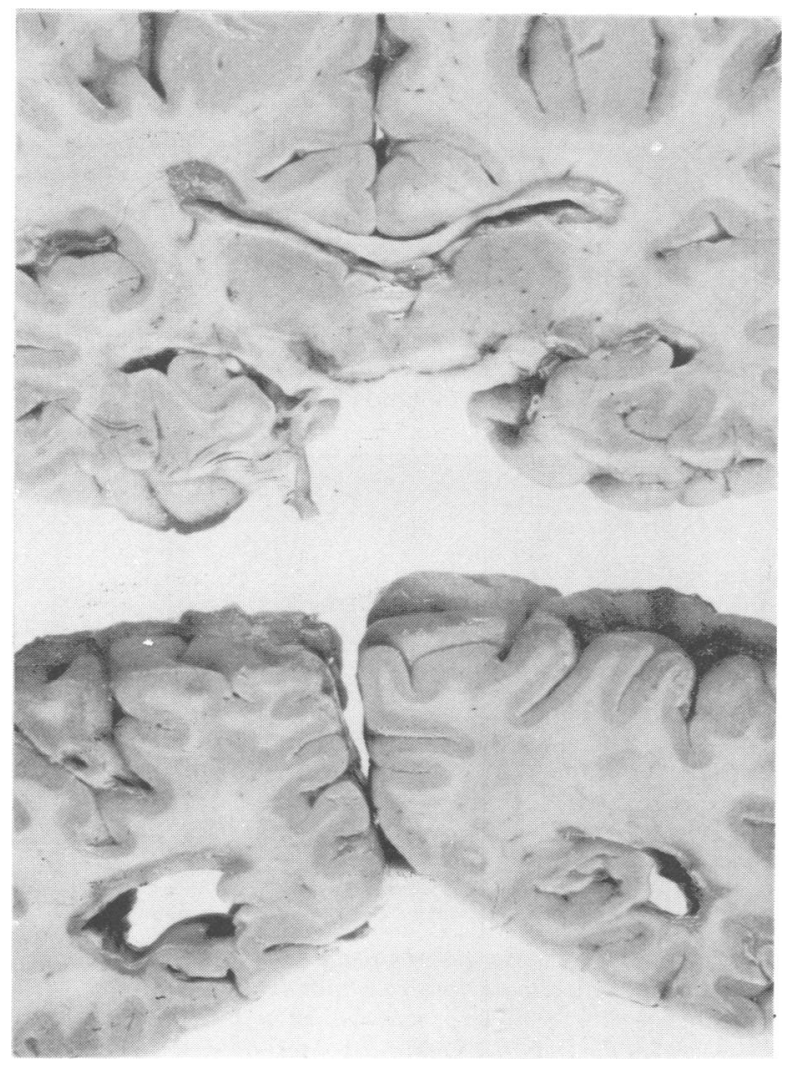

was noted to have severe flexion contractures of all four limbs, which apparently had developed secondary to prolonged immobility in bed, although involvement of the hands was difficult to explain on this basis. Complete neurological assessment was not possible due to poor cooperation but obvious wasting of small muscles of the hand and thigh muscles was noted, suggesting longstanding disuse. Investigations revealed that he had acute lymphoblastic leukaemia. He remained very ill, semicomatose and doubly incontinent and died within a week of admission. At necropsy the presence of acute lymphoblastic leukaemia with widespread deposits was confirmed. In the brain there were large periventricular plaques of demyelination (fig 1) mainly in the parietal and occipital lobes, but lesions were also found in the subcortical white matter and brain stem. Although progressive multifocal leucoencephalopathy is a known complication of leukaemia, this was excluded pathologically, the features being those of long-standing, inactive multiple sclerosis.

Group 2 Cases with a history of neurological illness, in whom a diagnosis of multiple sclerosis was never considered Case 5 A woman born in 1935 had a large right cerebral cystic tumour removed at the age of four years followed by radiotherapy. This had been carried out at another hospital and details were not available. Thereafter she remained
Fig 1 Case 4. Sections of the cerebral hemispheres showing paraventricular plaques of multiple sclerosis. 
mentally retarded, had fits, spastic left hemiplegia and post-papilloedematous optic atrophy. She was under continuous care in a mental subnormality unit and no new neurological features were noted. She died suddenly in 1975 following a short febrile illness with respiratory infection. At necropsy, apart from patchy bronchopneumonia, abnormalities were confined to the CNS. A large cavity filled with CSF was present in the right cerebral hemisphere at the site of the old surgical field. A few small scattered meningiomatous nodules were found in the occipital region on both sides and there was a small cerebellar cavernous haemangioma. The features were suggestive of the central form of Von Recklinghausen's disease and it seemed likely that the tumour removed in childhood was associated with this disorder. In addition there were large plaques of demyelination around the posterior parts of both lateral ventricles and scattered small, similar lesions were found in the brainstem and spinal cord (fig 2). Many of the plaques were far removed from the field where radiotherapy would most probably have been applied in 1939 and there were none of the vascular changes characteristic of irradiation damage (fig 2). Surviving axons could be demonstrated in demyelinated lesions (fig 3 ) and the overall features were considered to be those of multiple sclerosis.

Case 6 A woman born in 1914 suddenly developed a left hemiparesis in $\mathbf{1 9 7 0}$ and the presumed diagnosis was of a cerebrovascular incident. In 1974 she was admitted to a geriatric hospital for investigation of frequent falls and vertigo. She was confused, demented and was found to have a residual spastic left hemiparesis, dysarthria and bilateral cerebellar ataxia. The CSF IgG level was normal and the VDRL was negative. Over the following three months the dysarthria and hemiparesis improved to some extent but the dementia remained unchanged. At the age of 61 she suddenly developed double vision had a fall and at this stage she had a marked spastic weakness of all four limbs, exaggerated jaw jerk and extensor plantar responses. The diagnosis again was that of diffuse cerebrovascular disease with recent brainstem involvement. CAT scanning was not

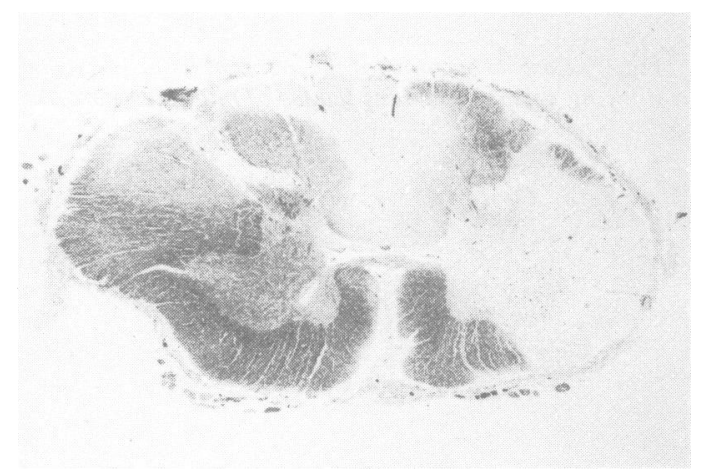

Fig 2 Case 5. Patches of demyelination in the spinal cord. Neurons survive in areas where plaques encroach on grey matter, and blood vessels are normal. (Luxol fast blue-cresyl violet, $\times 5 \cdot \therefore$.) available at the time. She remained demented and bedbound requiring continuous catheterisation till her death in 1975. At necropsy, apart from mild generalised atheroma, abnormalities were confined to the central nervous system, where widespread plaques of demyelination were found in the cerebral hemispheres and brainstem.

Case 7 A woman born in 1902 was admitted to a geriatric ward in October 1973 following an episode of loss of consciousness. On recovery from coma she was noted to be confused and had an extensor right plantar response. Her mental state improved gradually and the plantar response became flexor. Two months later she was found on the floor of her house. At this stage she had features of intellectual deterioration and was dysarthric. Two months later she had a series of grand mal fits during one day, resulting in a fall and a fractured neck of femur. After the fracture had been treated she continued to have fits and on one occasion was noted to have conjugate deviation of the eyes to the right before the seizure. The neurological illness was considered to be due to cerebrovascular disease. She died in 1974 due to bronchopneumonia. At necropsy, apart from bronchopneumonia, abnormalities were confined to the CNS. There were bilateral, sharply defined patches of demyelination, typical of multiple sclerosis, beside the lateral ventricular walls, especially the posterior horns. Lesions were not identified in the cerebellum, brain stem or optic pathways. The spinal cord was not examined. Numerous senile plaques were present in the hippocampal region, but there were only occasional neurofibrillary tangles.

Case 8 A man born in 1911 started in 1961 to suffer from episodic vertigo lasting a few hours each time. When first seen in 1966, he was found to have nystagmus on left lateral gaze and reduction of pinprick sensation over the left trigeminal 2nd division territory. CSF examination was normal, including a negative WR. No definite diagnosis was made. In 1969 he was referred again due to the development of intellectual deterioration and a short shuffling gait. On examination he was found to have bilat-

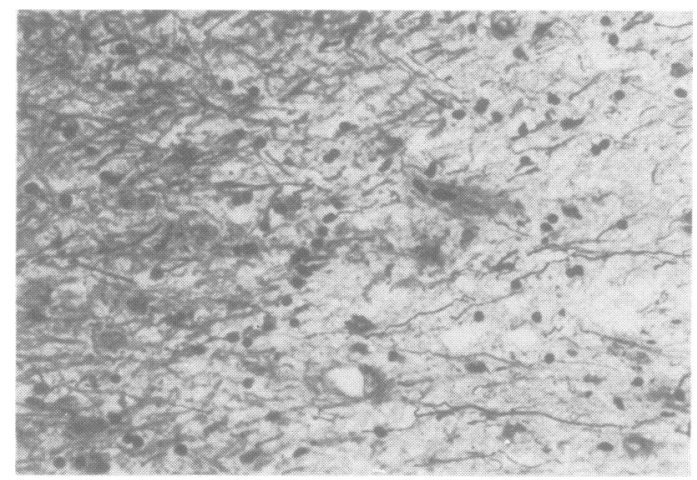

Fig 3 Case 5. Margin of a plaque in the cerebellar white matter stained to demonstrate both axons and myelin sheaths. Preserved axons are shown in the demyelinated area on the right. (Bodian-luxol fast blue, $\times 250$.) 
eral palmomental reflexes, positive glabellar tap, nystagmus on left lateral gaze, intellectual deterioration, possible left homonymous field defect, cogwheel rigidity of the left arm and both legs and extensor plantar responses. Sensory testing was unreliable. The clinical picture, including the late age of onset, early intellectual deterioration and extrapyramidal features, favoured a clinical diagnosis of diffuse cerebrovascular or system degenerative disease. He died in 1970 with myocardial infarction. At necropsy the major intracranial arteries showed moderate atheroma and there were small areas of old infarction in the basal ganglia and cerebellum. Patches of demyelination characteristic of multiple sclerosis were present beside the lateral ventricles, especially posteriorly, and in the brainstem.

Group 3 Cases with a history of neurological disorder in whom a diagnosis of multiple sclerosis was rejected or considered unlikely

Case 9 A woman aged 53 years suddenly developed a spastic right monoparesis with minimal impairment of vibration sense. Examination of the CSF and myelography were normal. The differential diagnosis was between cerebrovascular disease and a first episode of multiple sclerosis. She presented three years later with sudden onset of headache, vomiting, neck stiffness and dysphasia. The CSF was uniformly bloodstained and carotid angiography showed a left middle cerebral artery aneurysm. She became comatose and died on the day of admission. Discovery of the aneurysm led to the conclusion that it had probably also been responsible for the patient's initial monoparesis. At necropsy, apart from gross left ventricular hypertrophy, abnormalities found were confined to the CNS. There was moderate subarachnoid haemorrhage due to rupture of a small aneurysm situated near the trifurcation of the left middle cerebral artery and multiple infarcts were present in both cerebral hemispheres attributable to vasospasm. In addition there was a large plaque of demyelination beside the body of the left lateral ventricle near the trigone. Histological evidence of active progression could be seen at the edge of the lesion.

Case 10 A woman born in 1923 suddenly lost vision in both eyes in 1966 , but after nine months, acuity had improved to 6/12 and 6/18 in the right and left eyes respectively. Both optic discs were noted to be pale. Serum vita$\min B_{12}$, WR and skull radiographs were normal, and there was no family history of relevance. She presented again in 1969 with a history of gradual deterioration of vision over a few days. On examination acuity was reduced to $R 6 / 36$ and $L \quad 6 / 60$, and right central and left arcuate scotomata were noted. The clinical diagnosis was bilateral optic neuritis. She was referred to the Urology Department in 1976 with a history of urinary frequency and urgency for three years. Urine cultures were sterile and, on the basis of cystoscopic findings, urethral stricture was diagnosed. In spite of urethral dilatation her symptoms persisted and a cystometrogram showed the features of an "irritable bladder". In 1977 she developed hypertension and was treated with atenolol. This was soon followed by the onset of transient cold and numb feeling in the patient's hands, which was thought to be a side effect of atenolol: neurological examination was normal apart from persisting poor visual acuity in the left eye. In 1979 she reported haemoptysis and on investigation was shown to have a right bronchial carcinoma with widespread metastases, which led to her death in 1980. At necropsy the presence of carcinomatosis from a right bronchial primary was confirmed. The brain showed several circumscribed demyelinated plaques in the white matter around both lateral ventricles and in the pons. The optic nerves, chiasma and tracts were almost totally demyelinated.

Case 11 A woman born in 1905 developed left optic neuritis in 1956, but this recovered fully in a few months. She was treated for hypertension and coronary insufficiency in 1972. In 1973 she was found comatose at home and after recovery of consciousness she was found to have a right hemiparesis, dysphasia and rotatory nystagmus: ECG showed a massive recent myocardial infarction, VDRL was negative and CSF IgG was normal. Carotid angiography showed total occlusion of the right anterior cerebral artery but both internal carotid arteries were patent. Isotope brain scan revealed an area of increased density in the right frontal region. CT scan facilities and evoked response investigations were not available at that time. She was treated for recurrent urinary tract infections from 1973 to 1978 , for which no underlying cause was found, and she had tonic/clonic fits, including two episodes of status epilepticus. From 1975 to 1978 there was increasing intellectual deterioration, but no great change occurred in the right hemiparesis. A diagnosis of cerebrovascular disease was favoured despite the history of optic neuritis and rotatory nystagmus. She died in 1978. The general necropsy findings were massive pulmonary embolism from deep venous thrombosis, bronchopneumonia, left ventricular hypertrophy, old myocardial infarcts and chronic pyelonephritis. The brain showed moderate diffuse cerebral atrophy and prominent atheroma of the major arteries. There was an old infarct in the left medial frontal region. The left hippocampus was shrunken and gliotic and the right one showed more recent ischaemic damage. Extensive periventricular and perivascular plaques of multiple sclerosis were found in both cerebral hemispheres.

Case 12 A man born in 1910 was seen in 1963 in the Neurosurgical Department owing to the gradual onset of paraesthesiae and pain down both arms on coughing and subjective sensory disturbance over both hands. On examination he showed generalised hyperreflexia, mild spastic paraparesis and ataxic gait. A cervical myelogram showed total hold-up of myodil at C5-6 region, but the CSF was normal. He was followed up for nine months with a diagnosis of cervical spondylosis and, due to persistence of the above signs and symptoms, he had a decompressive cervical laminectomy. This was followed by marked improvement in walking. In 1974 he was referred to the Neurology Department with a history of progressive weakness and stiffness of the arms and legs and urinary incontinence. On examination he was found to have rotatory nystagmus, weakness of the right arm, spastic paraparesis and absent vibration sense below the iliac crest. A repeat myelogram showed some ligamentous thickening and minor disc protrusion at $\mathrm{C} 3-4$ level but no significant impingement on the cord. CSF examination showed ele- 
vated protein and normal IgG. Multiple sclerosis was considered in the differential diagnosis, but, even though the criteria outlined by Brain and Wilkinson ${ }^{14}$ were applied, no firm conclusion was reached. In 1976 he died with myocardial infarction. At necropsy pulmonary oedema and myocardial infarction were found. In the brain there were multiple grey and slightly yellowish patches of demyelination, many of them large, situated in the periventricular and subcortical white matter. Some of the lesions showed peripheral cellularity indicative of recent progression of the disease. A small recent infarct was noted in the right striatal region. In the spine a calcified projection at C3-4 level appeared to cause some indentation of the cord. Mild bilateral Wallerian degeneration in the pyramidal tracts was present at all levels in the spinal cord.

\section{Discussion}

The diagnosis of multiple sclerosis still is based essentially on clinical assessment of symptoms and signs, although it may be supported by the presence of elevated CSF IgG levels or periventricular lowdensity areas in CT scan. Prolonged latency of evoked responses, especially visual, provide evidence of a "second lesion" and aid early diagnosis.

Review of previously reported cases of unexpected detection of multiple sclerosis at necropsy reveals that 18 were clinically truly silent (Vost $e t$ $a l,{ }^{8}$ four cases, Russell ${ }^{7}$ three cases, Mackay and Hirano 9 two of nine cases, Koya et al $^{13}$ one case and Georgi $^{5}$ eight of 12 cases). Assessment of Georgi's ${ }^{5}$ report, however, is difficult owing to paucity of clinical information. An accepted important drawback for all studies of this type is that they are retrospective, relying substantially on clinical records. Minor or transient symptoms may be forgotten by the patient or neglected by the clinician. Ghatak and Hirano, ${ }^{11}$ for example, reported a 66-year-old man who had died of bronchial carcinoma and had a demyelinated plaque in the cervical cord: scrutiny of the medical records failed to reveal any note of previous neurological illness, but his wife later recalled that he had had an episode of leg weakness 30 years before he died.

In our first group of patients (Cases 1-4) there was no known previous history of neurological illness. Case 2, however, was an alcoholic living alone who had neglected himself, so that his account of previous ailments was not entirely reliable. Considered in retrospect, the initial paraesthesiae in the left leg in Case 4 could possibly be attributed to multiple sclerosis, but there were no abnormal physical signs.

The patients in our second group (Cases 5-8) did have a previous history of neurological or psychiatric disturbance, but multiple sclerosis was never considered in the differential diagnosis. Case
5 was severely mentally retarded following surgery for an intracranial tumour and, although her ability to give a history was limited, no change had been noted in her condition over the years. Cases 6,7 and $8 \mathrm{had}$ signs and symptoms of multiple lesions in the CNS but an acute onset over the age of 50 and early intellectual deterioration led to a diagnosis of cerebrovascular disease. Case 8 presented with paroxysmal vertigo and it is debatable whether this was in fact attributable to his multiple sclerosis. Twomey and Espir ${ }^{15}$ in their review of the literature did not find paroxysmal vertigo alone reported as a presenting feature of the disease, although they point out, that, "vertigo does occur in association with brainstem disturbances in multiple sclerosis".

Intellectual deterioration, which occurred in three of the patients in Group 2 and Case 11 from Group 3 , tends to be a misleading clinical feature in multiple sclerosis, especially in the elderly. Brain ${ }^{16}$ pointed out that mental symptomatology in multiple sclerosis had received little attention. Surridge ${ }^{17}$ noted an incidence of intellectual disorder as high as $61.1 \%$ in a group of 198 patients: 55 individuals had a score of more that III on McAlpine' $\mathrm{s}^{18}$ disability grading and, although he did not discuss disease duration, one suspects that dementia occurred mainly in patients with longstanding multiple sclerosis. All patients in his series were under the age of 40 and most other studies have been of the 20-50 age group. ${ }^{1920}$ Our cases 6, 8 and 11 developed intellectual deterioration at 4,8 and 19 years respectively after their initial neurological symptoms, which had arisen between the ages of 50 and 56 years. In Case 7 dementia was one of the presenting features at the age of 71 , but the brain did also show some changes of the Alzheimer disease type. The implication is that multiple sclerosis should be considered as a possible diagnosis when dementia occurs early in the course of any neurological disease with focal signs, regardless of the age of onset, especially if there is evidence of brainstem involvement.

The patients in our Group 3 (Cases 9-12) had a history of neurological disorder and, although a diagnosis of multiple sclerosis was considered at some stage, it was either rejected, regarded as unlikely or could not be established. In Case 9 the aneurysm, which became manifest by rupture shortly before death, was considered to be the likely cause of an isolated monoparesis 4 years earlier. Cases 10 and 11 presented with optic neuritis 4 years and 22 years before death respectively. The significance of isolated optic neuritis is always difficult to assess. Kurland $e t a l^{21}$ found that only $13 \%$ of 183 patients with the condition developed multiple sclerosis in the subsequent $12-18$ years and 
the corresponding figures in the investigation by Rose $e$ t $a^{22}$ were $58 \%$ of patients in a 32.7 month follow-up. Compston et $a^{23}$ found that in patients presenting with optic neuritis the incidence of subsequent multiple sclerosis was greater when there was a history of recurrent attacks than if just a single episode had occurred. In our Case 10 urinary symptoms were attributed to urethral stricture, but in spite of dilatation and repeated negative urine cultures, symptoms persisted. Such patients are usually initially referred to the urologist and in the presence of a local structural lesion, previous neurological history tends to be overlooked. We submit that a detailed past neurological history should be taken as part of a routine urological assessment. In Case 11 it was 17 years after an episode of optic neuritis when major neurological abnormalities arose, but at this stage she also had hypertension and ischaemic heart disease: a diagnosis of cerebrovascular disease was favoured and this was undoubtedly an important component of the neurological disorder, but, viewed with hindsight, the past history of optic neuritis and the occurrence of rotatory nystagmus indicative of a brainstem lesion were pointers to the presence of multiple sclerosis.

Multiple sclerosis with cervical cord involvement can be difficult to distinguish from cervical spondylotic myelopathy. The two conditions may even coexist and give rise to combined neurological disorder (Brain and Wilkinson ${ }^{14}$ ). Features favouring a diagnosis of cervical spondylosis in our Case 12 were pain down both arms, absence of abnormal neurological signs above the neck and complete obstruction to the flow of myodil in the cervical region. Wasting of the small muscles of the hands and inversion or diminution of reflexes in the arms, ${ }^{14}$ however, were absent. The patient's rapid improvement following laminectomy supported this assessment and indeed there was evidence of cervical spondylosis at necropsy. To what extent the early symptoms and signs could be attributed to either or both of the disorders is debatable.

Epileptiform attacks, which are uncommon in multiple sclerosis and may, therefore, be misleading in diagnosis, occurred in our Cases 7 and 11 . In the 1970 prevalence study in Aberdeen ${ }^{24} 1.8 \%$ of multiple sclerosis patients were found to have had an epileptic attack at some time in the course of the disease. An incidence of $1.5-4.5 \%$ has been reported in various other series. ${ }^{25-27}$ The mechanism of the genesis of fits in this disease has not been established. Matthews ${ }^{28}$ and Davis et al ${ }^{29}$ have suggested deficiency of ionised calcium as the basic factor underlying various paroxysmal disturbances in multiple sclerosis.

Approximately $20-25 \%$ of patients who die in hospital in the Grampian Region come to necropsy. In our study an average of one case of multiple sclerosis per year was discovered unexpectedly and this suggests that at least 4-5 patients each year may die with undetected disease in this region. Most of our patients, however, were investigated before the availability of computed tomography or the measurement of evoked responses and it is likely that fewer cases of multiple sclerosis with atypical presentation will escape detection in future. Some, nevertheless, will inevitably remain silent or undiagnosed, leading to an underestimate of disease prevalence.

We are grateful to $\mathrm{Dr}$ AW Downie for helpful advice.

\section{References}

${ }^{1}$ Karplus JP. Organische, nichttraumatische Nervenkrankheiten bei Kriegsteilnehmern. Wein Med Wochenschr 1919;69:137-48.

${ }^{2}$ Reuter A, Gaupp R Jr. Beitrag zur Frage der akuten multiplen Sklerose. Z Ges Neurol Psychiat 1932; 138:495-502.

${ }^{3}$ Friedman AP, Davison C. Multiple sclerosis with late onset of symptoms. Arch Neurol Psychiat 1945; 54:348-60.

${ }^{4}$ Daum S, Vogein-Rappoport M, Gruner J, Foncin JF. Sclérose en plaque révélée a l'âge de 64 ans par une névralgie trigeminale: étude anatamo-clinique. $R e v$ Neurol (Paris) 1960;102:500-3.

${ }^{5}$ Georgi W. Multiple Sklerose: Pathologisch-anatomische Befunde multipler Sklerose bei klinisch nicht diagnostizierten Krankheiten. Schweiz Med Wochenschr 1961;91:605-7.

${ }^{6}$ McLardy T, Sinclair A. A case of presenile fulminating multiple sclerosis (with pathogenic considerations). Confin Neurol 1964;24:417-24.

${ }^{7}$ Russell DS. Trauma and multiple sclerosis. Lancet 1964;1:978.

${ }^{8}$ Vost A, Wolochow DA, Howell DA. Incidence of infarcts of the brain in heart disease. $J$ Pathol Bact 1964;88:463-70

${ }^{9}$ Mackay RP, Hirano A. Forms of benign multiple sclerosis; report of two "clinically silent" cases discovered at autopsy. Arch Neurol 1967;17:588-600.

${ }^{10}$ Fog T, Hyllested K, Andersen SR. A case of benign multiple sclerosis, with autopsy. Acta Neurol Scand 1972;48 (Suppl 51):369-70.

$"$ Ghatak NR, Hirano A, Lijtmaer H, Zimmerman HM. Asymptomatic demyelinated plaque in the spinal cord. Arch Neurol 1974;30:484-6.

12 Weber W, Ulrich J. Multiple sclerosis, diffuse lymphoplasmacytic encephalitis and Alzheimer's disease in a patient with progressive dementia. Eur Neurol 1976;14:266-74. 
${ }^{13}$ Koya G, Suzuki M, Baba M, Iwabuchi T. An autopsy case of concurrence of multiple sclerosis and glioma. Hirosaki Med J 1977;29:588-96.

${ }^{14}$ Brain WR, Wilkinson M. The association of cervical spondylosis and disseminated sclerosis. Brain 1957;80:456-78.

15 Twomey JA, Espir MLE. Paroxysmal symptoms as the first manifestations of multiple sclerosis. J Neurol Neurosurg Psychiatry 1980;43:296-304.

${ }^{16}$ Brain WR. Critical Review: disseminated sclerosis. QJ Med 1930;23:343-91.

${ }^{17}$ Surridge D. An investigation into some psychiatric aspects of multiple sclerosis. $\mathrm{Br} J$ Psychiatry 1969;115:749-64.

${ }^{18}$ McAlpine D, Lumsden CE, Acheson ED. Multiple Sclerosis. A Reappraisal. 2nd ed. London: Churchill Livingstone, 1972:229-32.

${ }^{19}$ Bergin JD. Rapidly progressing dementia in disseminated sclerosis. J Neurol Neurosurg Psychiatry 1957;20:285-92.

${ }^{20}$ Young AC, Saunders J, Ponsford JR. Mental change as an early feature of multiple sclerosis. J Neurol Neurosurg Psychiatry 1976;39:1008-13.

${ }^{21}$ Kurland LT, Auth TL, Beebe GW, Kurtzke JF, Lessell $S$, Nagler B, Nefzger MD. Studies on the natural history of multiple sclerosis. II: Progression from optic neuropathy to multiple sclerosis. Trans Am Neurol Assoc 1963;88:231-5.

${ }^{22}$ Rose FC, Friedman AI, Bowden PMA, Perkin GD. In: McAlpine D, Lumsden CE, Acheson ED, eds. Multiple Sclerosis. A Reappraisal. 2nd ed. London: Churchill Livingstone, 1972:151-2.

${ }^{23}$ Compston DAS, Batchelor JR, Earl CJ, McDonald WI. Factors influencing the risk of multiple sclerosis developing in patients with optic neuritis. Brain 1978;101:495-511.

${ }^{24}$ Shepherd DI. Multiple sclerosis in North-East Scotland. MD thesis, University of Aberdeen 1976; Vol 1: 134.

${ }^{25}$ Matthews WB. Epilepsy and disseminated sclerosis. $Q J$ Med 1962;31:141-55.

${ }^{26}$ Müller R. Studies in disseminated sclerosis. Acta Med Scand 1949;Suppl 222: 86.

${ }^{27}$ Drake WE, Macrae D. Epilepsy in multiple sclerosis. Neurology (Minneap) 1961;11:810-6.

${ }^{28}$ Matthews WB. Paroxysmal symptoms in multiple sclerosis. J Neurol Neurosurg Psychiatry 1975; 38: 617-23.

${ }^{29}$ Davis FA, Becker FO, Michael JA, Sorensen E. Effects of intravenous sodium bicarbonate, disodium edetate $\left(\mathrm{Na}_{2}\right.$ EDTA), and hyperventilation on visual and oculomotor signs in multiple sclerosis. J Neurol Neurosurg Psychiatry 1970;33:723-32. 